\title{
Sarcopenia may Influence the Prognosis in Advanced Thyroid Cancer Patients Treated With Molecular Targeted Therapy
}

\author{
AKIHIRO NISHIYAMA ${ }^{1}$, YUKIKO STAUB ${ }^{2}$, YUKIO SUGA ${ }^{2}$, MIKIYA FUJTA ${ }^{2}$, \\ AZUSA TANIMOTO $^{1}$, KOUSHIRO OHTSUBO $^{1}$ and SEIJI YANO ${ }^{1}$ \\ ${ }^{1}$ Division of Medical Oncology, Cancer Research Institute, Kanazawa University, Kanazawa, Japan; \\ ${ }^{2}$ Department of Clinical Pharmacy and Healthcare Sciences, Faculty of Pharmacy, Institute of Medical, \\ Pharmaceutical and Health Sciences, Kanazawa University, Kanazawa, Japan
}

\begin{abstract}
Background/Aim: Reportedly, sarcopenia and nutritional status are associated with prognosis in cancer patients. However, data regarding the relationship of these factors with advanced thyroid cancer patients receiving molecular targeted therapy remains scarce. Therefore, we investigated the relationship between nutritional assessment, as well as sarcopenia, and prognosis in patients with advanced thyroid cancer undergoing molecular targeted therapy. Patients and Methods: In this retrospective study, sarcopenia and several markers of nutritional status were assessed in advanced thyroid cancer patients at the Kanazawa University Hospital, before the introduction of molecular targeted therapy. Results: Advanced thyroid cancer patients with sarcopenia presented a worse prognosis than those without sarcopenia. Additionally, sarcopenia strongly correlated with several markers of nutritional status, such as albumin, prognostic nutrition index, and Glasgow prognostic score. Conclusion: Sarcopenia could be a prognostic factor in patients with advanced thyroid cancer receiving molecular targeted therapy.
\end{abstract}

The Ministry of Environment of Japan reported that the incidence of thyroid cancer in Japan is growing annually, and especially in women, it rose from about 3 per 100,000 individuals in 1975 to more than 13 per 100,000 individuals in 2013 (1). Thyroid cancer is categorized into: i) differentiated thyroid carcinoma (DTC), consisting of papillary thyroid

This article is freely accessible online.

Correspondence to: Akihiro Nishiyama, Division of Medical Oncology, Cancer Research Institute, Kanazawa University, 13-1, Takaramachi, Kanazawa, Ishikawa 920-0934, Japan. Tel: +81 762652794, Fax: +81 762442454, e-mail: an0510@staff.kanazawau.ac.jp

Key Words: Sarcopenia, nutritional assessment, advanced thyroid cancer patient, molecular targeted therapy. carcinoma (PTC) and follicular thyroid carcinoma (FTC), ii) medullary thyroid carcinoma (MTC), and iii) anaplastic thyroid carcinoma (ATC). Although most patients with DTC demonstrate a good prognosis, some present aggressive features (2). Recently, two tyrosine kinase inhibitors (TKI), sorafenib and lenvatinib, were approved for radioiodine-refractory DTC by the Food and Drug Administration (FDA), as an add-on to standard classical therapy, including surgery, radioactive iodine (RAI), and thyroid-stimulating hormone (TSH) suppression therapy (3, 4). These two drugs, presenting different adverse effect profiles, have resulted in prolonged progression-free survival (PFS). Conversely, ATC is one of the most aggressive solid tumors observed in humans (5), frequently originating in a pre-existing thyroid cancer lesion, as proposed by the simultaneous occurrence of areas DTC and poorly DTC (6). In Japan, based on phase II trial results, lenvatinib has been approved for the treatment of ATC (7). However, when clinicians encounter RAI refractory DTC or ATC, they must combat advanced thyroid cancer with limited treatment options.

In cancer patients, nutritional management during therapy remains crucial. As their nutritional status is related to prognosis, clinicians should be aware of nutritional assessment methods, and use them carefully in clinical practice (8). Several laboratory nutritional markers, including serum albumin (Alb), C-reactive protein (CRP), and total lymphocyte count, have been frequently used by physicians to assess patients' nutritional status. Sarcopenia is a severe depletion of skeletal muscle and has been evaluated in cancer patients as it has been linked with the incidence of toxicity and bad prognosis, indicating poor overall survival or high risk of postoperative complications ( 9 , 10). Despite these links, data regarding nutrition assessment and sarcopenia in thyroid cancer remains limited. Here, we speculated that it could be crucial to assess a patients' nutritional status before treatment, allowing the safe use of molecular targeted therapy. In this study, we investigated the relationship between nutritional assessment, as well as sarcopenia, and the prognosis of advanced thyroid cancer patients treated with lenvatinib or sorafenib at the Kanazawa University Hospital. 
Table I. Patients' basic characteristics.

\begin{tabular}{|c|c|c|c|c|}
\hline \multirow[t]{2}{*}{ Characteristics } & & \multicolumn{2}{|c|}{ Median (range) } & \multirow[t]{2}{*}{$p$-Value } \\
\hline & & $\begin{array}{l}\text { Sarcopenia } \\
\quad(\mathrm{n}=9)\end{array}$ & $\begin{array}{c}\text { Non-sarcopenia } \\
\quad(\mathrm{n}=14)\end{array}$ & \\
\hline Age, years & & $67(49-86)$ & $67(45-77)$ & $0.614^{\mathrm{h}}$ \\
\hline \multirow[t]{2}{*}{ Gender, n (\%) } & Male & $4(44.4)$ & $9(64.3)$ & $0.417^{\mathrm{i}}$ \\
\hline & Female & $5(55.6)$ & $5(35.7)$ & \\
\hline Height, $\mathrm{cm}$ & & $158(145-168)$ & $163(147-181)$ & $0.201^{\mathrm{h}}$ \\
\hline Body weight, $\mathrm{kg}$ & & $51.9(29.5-60.9)$ & $64.3(44.9-90.6)$ & $0.003^{\mathrm{h}}$ \\
\hline Body surface area, $\mathrm{m}^{2}$ & & $1.53(1.11-1.69)$ & $1.72(1.41-2.06)$ & $0.009^{\mathrm{h}}$ \\
\hline Body mass index, $\mathrm{kg} / \mathrm{m}^{2}$ & & $20.0(14.1-24.8)$ & $23.8(18.3-37.5)$ & $0.007^{\mathrm{h}}$ \\
\hline \multirow[t]{2}{*}{ Skeletal mass index, $\mathrm{kg} / \mathrm{m}^{2}$} & Male & $34.2(29.6-38.9)$ & $50.8(41.5-56.3)$ & $0.003^{\mathrm{h}}$ \\
\hline & Female & $27.8(23.1-28.9)$ & $39.9(32.0-42.8)$ & $0.008^{\mathrm{h}}$ \\
\hline \multirow[t]{4}{*}{ ECOG PSa, n (\%) } & 0 & $0(0)$ & $5(35.7)$ & $0.010^{\mathrm{h}}$ \\
\hline & 1 & $5(55.6)$ & $8(57.1)$ & \\
\hline & 2 & $1(11.1)$ & $1(7.1)$ & \\
\hline & 3 & $3(33.3)$ & $0(0)$ & \\
\hline \multirow[t]{2}{*}{ TKI treatment ${ }^{\mathrm{b}}, \mathrm{n}(\%)$} & Lenvatinib & $8(88.9)$ & $11(78.6)^{\mathrm{c}}$ & $1.000^{\mathrm{i}}$ \\
\hline & Sorafenib & $1(11.1)$ & $6(42.9)^{\mathrm{c}}$ & $0.176^{\mathrm{i}}$ \\
\hline \multirow[t]{4}{*}{ Histological type, n (\%) } & Papillary & $4(44.4)$ & $6(42.9)$ & $1.000^{\mathrm{i}}$ \\
\hline & Follicular & $1(11.1)$ & $3(21.4)$ & $1.000^{\mathrm{i}}$ \\
\hline & Poorly differentiated & $1(11.1)$ & $3(21.4)$ & $1.000^{\mathrm{i}}$ \\
\hline & Anaplastic & $3(33.3)$ & $2(14.3)$ & $0.343^{\mathrm{i}}$ \\
\hline \multirow[t]{4}{*}{ Prior treatment, $\mathrm{n}(\%)$} & Surgery & $5(55.6)$ & $12(85.7)$ & $0.343^{\mathrm{i}}$ \\
\hline & Radioiodine & $2(22.2)$ & $9(64.3)$ & $0.089^{\mathrm{i}}$ \\
\hline & External radiation & $4(44.4)$ & $5(35.7)$ & $1.000^{\mathrm{i}}$ \\
\hline & Cytotoxic agents & $3(33.3)$ & $0(0)$ & $0.047^{\mathrm{i}}$ \\
\hline \multirow[t]{9}{*}{ Target lesions, n (\%) } & Any & $8(88.9)$ & $14(100)$ & \\
\hline & Lymph node & $5(55.6)$ & $8(57.1)$ & $1.000^{\mathrm{i}}$ \\
\hline & Lung & $2(22.2)$ & $6(42.9)$ & $0.400^{\mathrm{i}}$ \\
\hline & Thyroid & $3(33.3)$ & $2(14.3)$ & $0.343^{\mathrm{i}}$ \\
\hline & Esophagus & $2(22.2)$ & $0(0)$ & $0.142^{\mathrm{i}}$ \\
\hline & Trachea & $0(0)$ & $2(14.3)$ & $0.502^{\mathrm{i}}$ \\
\hline & Liver & $1(11.1)$ & $1(7.1)$ & $1.000^{\mathrm{i}}$ \\
\hline & Bone & $2(22.2)$ & $1(7.1)$ & $0.538^{\mathrm{i}}$ \\
\hline & Brain & $1(11.1)$ & $1(7.1)$ & $1.000^{\mathrm{i}}$ \\
\hline \multirow[t]{5}{*}{ Tumor invasion, $\mathrm{n}(\%)$} & Any & $5(55.6)$ & $4(28.6)$ & $0.383^{\mathrm{i}}$ \\
\hline & Vessel & $4(44.4)$ & $3(21.4)$ & $0.363^{\mathrm{i}}$ \\
\hline & Esophagus & $2(22.2)$ & $0(0)$ & $0.142^{\mathrm{i}}$ \\
\hline & Trachea & $0(0)$ & $1(7.1)$ & $1.000^{\mathrm{i}}$ \\
\hline & Skin & $1(11.1)$ & $1(7.1)$ & $1.000^{\mathrm{i}}$ \\
\hline Baseline tumor size, $\mathrm{mm}$ & & $83.2^{\mathrm{d}}(40.5-178.1)$ & $51.0(11.5-101.8)$ & $0.042^{\mathrm{h}}$ \\
\hline Baseline thyroglobulin, $\mathrm{ng} / \mathrm{mL}$ & & $1041.4^{\mathrm{e}}(5.3-273,440)$ & $334.4(2.6-19,837)$ & $0.664^{\mathrm{h}}$ \\
\hline anti-thyroglobulin antibody & $\geq 28.0 \mathrm{IU} / \mathrm{ml}, \mathrm{n}(\%)$ & $3(37.5)^{\mathrm{e}}$ & $4(33.3)^{\mathrm{f}}$ & $1.000^{\mathrm{i}}$ \\
\hline
\end{tabular}

aECOG: Eastern Cooperative Oncology Group performance status; bTKI: tyrosine kinase inhibitors; ${ }^{\mathrm{C}}$ Two patients in the non-sarcopenia group received lenvatinib after sorafenib. The reasons for switching TKI was adverse effects in one patient, and serum thyroglobulin increase in the other patient. One patient in the non-sarcopenia group received sorafenib after lenvatinib because of adverse effects; ${ }_{\mathrm{n}}=8$ patients with at least one

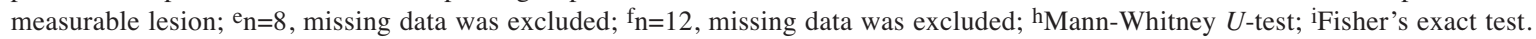

\section{Patients and Methods}

Patients and study design. In this retrospective study, patients treated for advanced thyroid cancer using lenvatinib, sorafenib, or both drugs interchanged, were selected from June 2014 to January 2019 at the Kanazawa University Hospital.
Measurement of muscle mass. As described previously, sarcopenia is determined according to the method briefly described below (11-13). After cross-sectional computed tomography (CT) imaging at the caudal end of the third lumbar vertebra level, skeletal muscle was measured, and skeletal muscle areas, including the psoas, erector spinae, quadratus lumborum, 

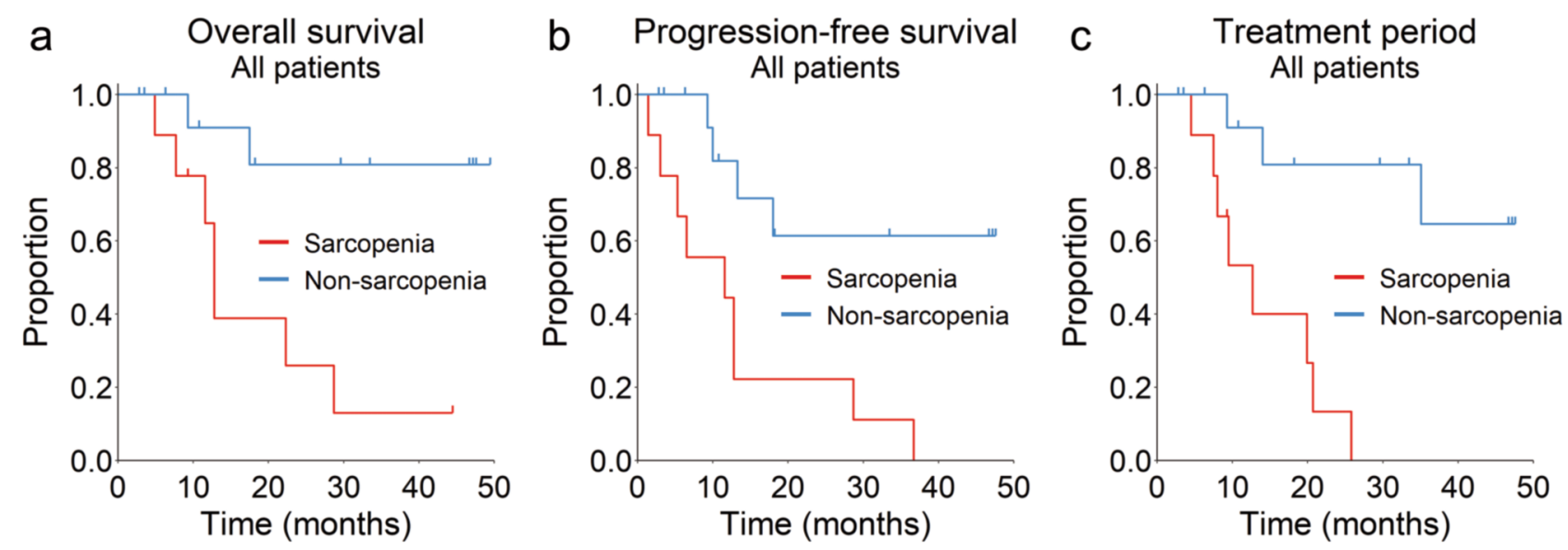

Figure 1. Overall survival (OS), progression-free survival (PFS), and treatment time (TP) for all patients with advanced thyroid cancer treated with molecular targeted therapy. The Kaplan-Meier curves estimating $O S(a), P F S(b)$, and TP (c) in 23 patients with advanced thyroid cancer.

Table II. Survival analysis between SG and NSG.

\begin{tabular}{|c|c|c|c|c|c|c|c|c|c|c|}
\hline \multirow[t]{2}{*}{ Survival time } & \multicolumn{5}{|c|}{ All patients $(n=23)$} & \multicolumn{5}{|c|}{ DTC patients $(\mathrm{n}=18)$} \\
\hline & $\mathrm{n}$ & $\begin{array}{c}\text { Median } \\
\text { (months) }\end{array}$ & $\begin{array}{l}95 \% \mathrm{CI} \\
\text { Lower }\end{array}$ & $\begin{array}{l}95 \% \mathrm{CI} \\
\text { Upper }\end{array}$ & $\begin{array}{c}p \text {-Value } \\
\text { (log-rank) }\end{array}$ & $\mathrm{n}$ & $\begin{array}{c}\text { Median } \\
\text { (months) }\end{array}$ & $\begin{array}{l}95 \% \text { CI } \\
\text { Lower }\end{array}$ & $\begin{array}{l}95 \% \text { CI } \\
\text { Upper }\end{array}$ & $\begin{array}{c}p \text {-Value } \\
\text { (log-rank) }\end{array}$ \\
\hline \multicolumn{11}{|l|}{ OS } \\
\hline All & 23 & 28.7 & 12.8 & NR & - & 18 & NR & 12.8 & NR & - \\
\hline Sarcopenia & 9 & 12.8 & 4.9 & 28.7 & 0.006 & 6 & 17.6 & 11.6 & NR & 0.005 \\
\hline Non-sarcopenia & 14 & NR & 17.5 & NR & & 12 & NR & 17.5 & NR & \\
\hline \multicolumn{11}{|l|}{ PFS } \\
\hline All & 23 & 13.3 & 10.0 & NR & - & 18 & 28.7 & 12.8 & NR & - \\
\hline Sarcopenia & 9 & 11.6 & 1.4 & 28.7 & 0.003 & 6 & 12.8 & 1.4 & NR & 0.001 \\
\hline Non-sarcopenia & 14 & NR & 10.0 & NR & & 12 & NR & 10.0 & NR & \\
\hline \multicolumn{11}{|l|}{ TP } \\
\hline All & 23 & 25.8 & 9.5 & NR & - & 18 & 35.1 & 14.0 & NR & - \\
\hline Sarcopenia & 9 & 12.7 & 4.5 & 20.7 & 0.001 & 6 & 11.1 & 3.3 & NR & $<0.001$ \\
\hline Non-sarcopenia & 14 & NR & 14.0 & NR & & 12 & NR & 14.0 & NR & \\
\hline
\end{tabular}

95\% CI: 95\% Confidence interval; DTC: differentiated thyroid carcinoma; OS: overall survival; PFS: progression-free survival; TP: treatment period; NR: not reached.

transversus abdominis, external and internal obliques, and rectus abdominis, were identified and quantified using -29 to 150 Hounsfield units (HU). The skeletal mass index (SMI) was calculated by normalizing these skeletal muscle areas for height $\left(\mathrm{cm}^{2} / \mathrm{m}^{2}\right)$. The cut-off values of SMI were set at $40.31 \mathrm{~cm}^{2} / \mathrm{m}^{2}$ for men and $30.88 \mathrm{~cm}^{2} / \mathrm{m}^{2}$ for women (11). Based on the SMI, patients were divided into either the sarcopenia group (SG) or the non-sarcopenia group (NSG). SG presented an SMI value lower than the cut-off value. For each patient, we assessed the SMI before molecular targeted therapy.

Nutritional assessment. In this study, we used several classical nutritional assessments, including body mass index (BMI), the Glasgow prognostic score (GPS), prognostic nutrition index (PNI), neutrophil-to-lymphocyte ratio (NLR), platelet-to-lymphocyte ratio
(PLR), and CRP-albumin ratio (CAR),. BMI was calculated as weight in kilograms divided by height in meters squared. GPS was evaluated as follows: patients with an elevated level of both CRP $(\mathrm{CRP}>1.0 \mathrm{mg} / \mathrm{dl})$ and hypoalbuminemia $(\mathrm{Alb}<3.5 \mathrm{~g} / \mathrm{dl})$ were allocated a score of 2 , and patients presenting one or none of these blood chemistry abnormalities were allocated a score 1 or 0 , respectively (14). PNI was calculated as $10 \times$ serum albumin value $(\mathrm{g} / \mathrm{dl})+0.005 \times$ peripheral lymphocyte count $\left(\right.$ per $\left.\mathrm{mm}^{3}\right)(15)$. NLR was calculated as the absolute neutrophil count divided by the absolute lymphocyte count (16). PNR was calculated as the absolute platelet count divided by the absolute lymphocyte count (17). CAR was calculated by dividing the serum CRP level $(\mathrm{mg} / \mathrm{dl})$ by the serum albumin level (g/dl) (18). For each patient, we determined these nutritional assessments before the initiation of molecular targeted therapy. 

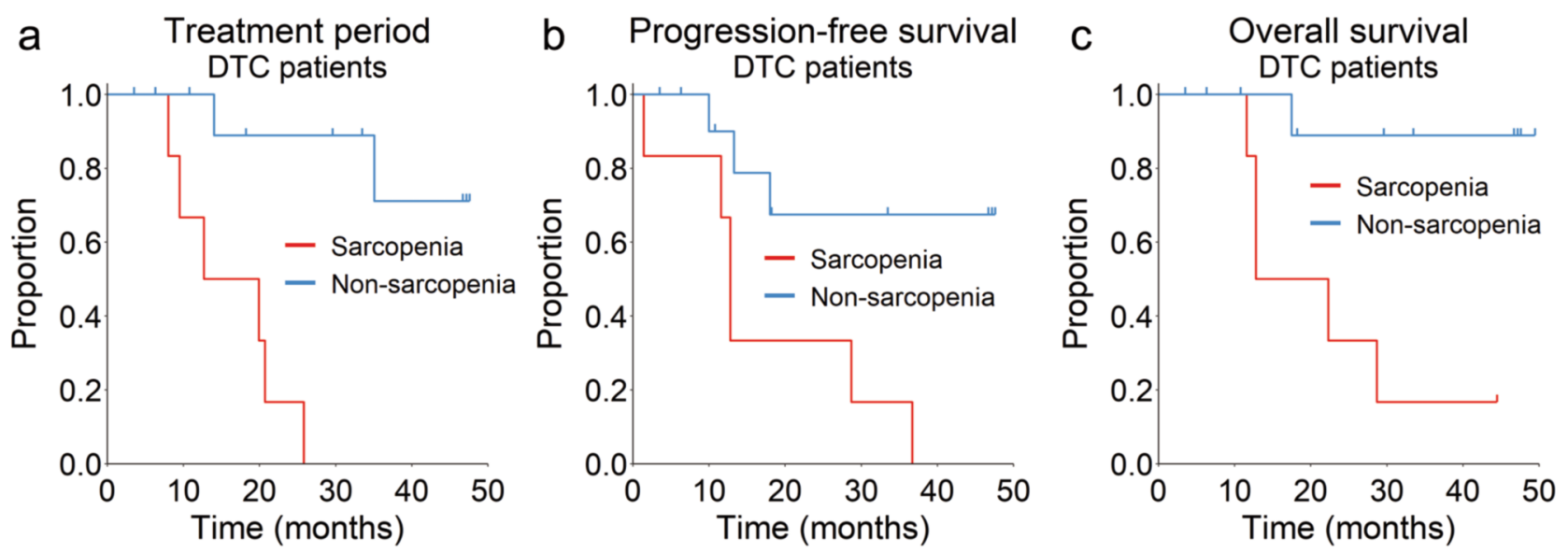

Figure 2. Overall survival (OS), progression-free survival (PFS), and treatment time (TP) for differentiated thyroid cancer patients treated with molecular targeted therapy. The Kaplan-Meier curves estimating OS (a), PFS (b), and TP (c) in 18 differentiated thyroid cancer patients.

Table III. Objective response.

\begin{tabular}{|c|c|c|c|c|c|c|c|c|c|c|}
\hline \multirow[t]{2}{*}{ Objective response } & \multicolumn{5}{|c|}{ All patients $(n=23)$} & \multicolumn{5}{|c|}{ DTC patients $(\mathrm{n}=18)$} \\
\hline & $\mathrm{n}$ & $\begin{array}{c}\mathrm{RR} \\
\mathrm{n}(\%)\end{array}$ & $\begin{array}{l}p \text {-Value } \\
\text { (Fisher) }\end{array}$ & $\begin{array}{l}\text { DCR } \\
\mathrm{n}(\%)\end{array}$ & $\begin{array}{l}p \text {-Value } \\
\text { (Fisher) }\end{array}$ & $\mathrm{n}$ & $\begin{array}{c}\mathrm{RR} \\
\mathrm{n}(\%)\end{array}$ & $\begin{array}{l}p \text {-Value } \\
\text { (Fisher) }\end{array}$ & $\begin{array}{l}\text { DCR } \\
\mathrm{n}(\%)\end{array}$ & $\begin{array}{l}p \text {-Value } \\
\text { (Fisher) }\end{array}$ \\
\hline All & 23 & $13(56.5)$ & - & $22(95.7)$ & - & 18 & $12(66.7)$ & - & $17(94.4)$ & - \\
\hline Sarcopenia & 9 & $4(44.4)$ & 0.417 & $8(88.9)$ & 0.391 & 6 & $3(50.0)$ & 0.344 & $5(83.3)$ & 0.333 \\
\hline Non-sarcopenia & 14 & $9(64.3)$ & & $14(100)$ & & 12 & $9(75.0)$ & & $12(100)$ & \\
\hline
\end{tabular}

DTC: Differentiated thyroid carcinoma; RR: response rate; DCR: disease control rate.

Efficacy of molecular targeted therapy. Using Computed Tomography imaging, objective tumor responses were assessed according to the Response Evaluation Criteria in Solid Tumors (RECIST), version 1.1 (19). We analyzed efficacy of molecular targeted therapy in all patients as well as in a subgroup of patients presenting DTC (DTC patients) alone. Firstly, these patients were divided into SG and NSG. Secondly, we conducted survival analyses and assessed the response rate (RR) as well as the disease control rate (DCR). Lastly, we analyzed survival time based on nutrition indexes, other than sarcopenia, for all patients and DTC patients.

Safety. Toxicities were evaluated in accordance with the National Cancer Institute Common Terminology Criteria for Adverse Events (CTCAE) version 5.0 (20). For common adverse events (AE), we assessed the grade of AE, while for severe adverse events (SAE), we assessed their frequency.

Statistics. Statistical analyses were performed using the R software version 3.5.1 (the R Foundation for Statistical Computing, Vienna, Austria). For survival analyses, the initial date of reckoning was defined as the start day for molecular targeted therapy. If patients received both lenvatinib and sorafenib, the initial date of reckoning was defined as the first day of either drug, whichever was administered first. Overall survival (OS) was defined as the time from the start of molecular targeted therapy until death owing to any cause. PFS was defined as the time from the start of molecular targeted therapy until the date of the first documentation of disease progression or death owing to any cause. Treatment period (TP) was defined as the time from the start of molecular targeted therapy to disease progression, death owing to any cause, or treatment cessation. Survival curves (OS, PFS, and TP) for SG and NSG were estimated using the Kaplan-Meier method and compared using the stratified log-rank test. Moreover, the impacts of sarcopenia and other nutritional markers were evaluated using the Cox proportional hazard model. Quantitative and ordinal variables, such as grades of $\mathrm{AE}$ between the two groups were non-parametrically analyzed using the Mann-Whitney $U$-test with box whisker plots. The lengths of the boxes referred to the interquartile range (IQR), with the middle lines representing the median values in each group. The upper whiskers referred to the largest observations no more than upper hinges+1.5×IQR, and the lower whiskers referred to the smallest observations no less than lower hinge-1.5×IQR. Outliers in data, beyond the end of the whiskers, were plotted individually. The ratio difference between groups was analyzed using Fisher's exact test. The correlation between sarcopenia and other nutritional markers was determined using Spearman's rank correlation coefficient, described graphically in a correlation matrix.

The scatter plot matrix is presented using bivariate scatter plots below the diagonal, histograms on the diagonal, and the Spearman's rank correlation coefficient above the diagonal. SARC, sarcopenia 
Nishiyama et al: Relationship Between Sarcopenia and Advanced Thyroid Cancer Patients

Table IV. Hazard ratios of baseline nutritional markers based on Cox proportional hazard model (univariate analyses).

\begin{tabular}{|c|c|c|c|c|c|c|c|c|}
\hline \multirow{2}{*}{$\begin{array}{l}\text { Cox proportional } \\
\text { hazard model }\end{array}$} & \multicolumn{4}{|c|}{ All patients $(n=23)$} & \multicolumn{4}{|c|}{ DTC patients $(\mathrm{n}=18)$} \\
\hline & HR & $\begin{array}{l}95 \% \mathrm{CI} \\
\text { Lower }\end{array}$ & $\begin{array}{l}95 \% \text { CI } \\
\text { Upper }\end{array}$ & $p$-Value & HR & $\begin{array}{l}95 \% \mathrm{CI} \\
\text { Lower }\end{array}$ & $\begin{array}{l}95 \% \mathrm{CI} \\
\text { Upper }\end{array}$ & $p$-Value \\
\hline \multicolumn{9}{|l|}{ OS } \\
\hline Sarcopenia & 6.892 & 1.409 & 33.710 & 0.017 & 11.43 & 1.317 & 99.21 & 0.027 \\
\hline BMI & 0.954 & 0.833 & 1.093 & 0.496 & 0.973 & 0.828 & 1.143 & 0.734 \\
\hline Alb & 0.227 & 0.068 & 0.758 & 0.016 & 0.075 & 0.010 & 0.549 & 0.011 \\
\hline PNI & 0.905 & 0.821 & 0.998 & 0.045 & 0.852 & 0.743 & 0.978 & 0.023 \\
\hline GPS & 2.342 & 1.124 & 4.880 & 0.023 & 3.674 & 1.358 & 9.939 & 0.010 \\
\hline NLR & 1.106 & 0.925 & 1.323 & 0.270 & 1.180 & 0.960 & 1.449 & 0.115 \\
\hline PLR & 1.001 & 0.998 & 1.004 & 0.513 & 1.002 & 0.998 & 1.005 & 0.292 \\
\hline CAR & 3.604 & 1.024 & 12.69 & 0.046 & 8.265 & 1.378 & 49.56 & 0.021 \\
\hline \multicolumn{9}{|l|}{ PFS } \\
\hline Sarcopenia & 5.084 & 1.543 & 16.75 & 0.008 & 5.050 & 1.242 & 20.53 & 0.024 \\
\hline BMI & 0.921 & 0.820 & 1.035 & 0.166 & 0.954 & 0.835 & 1.089 & 0.482 \\
\hline Alb & 0.232 & 0.085 & 0.634 & 0.004 & 0.180 & 0.050 & 0.653 & 0.009 \\
\hline PNI & 0.910 & 0.842 & 0.983 & 0.017 & 0.901 & 0.820 & 0.990 & 0.030 \\
\hline GPS & 2.249 & 1.186 & 4.268 & 0.013 & 2.527 & 1.161 & 5.499 & 0.019 \\
\hline NLR & 1.109 & 0.956 & 1.285 & 0.171 & 1.135 & 0.954 & 1.350 & 0.153 \\
\hline PLR & 1.001 & 0.999 & 1.003 & 0.378 & 1.001 & 0.998 & 1.004 & 0.372 \\
\hline CAR & 3.286 & 1.151 & 9.385 & 0.026 & 3.332 & 0.791 & 14.04 & 0.101 \\
\hline \multicolumn{9}{|l|}{$\mathrm{TP}$} \\
\hline Sarcopenia & 9.808 & 2.010 & 47.870 & 0.005 & 17.92 & 2.087 & 153.900 & 0.009 \\
\hline BMI & 0.945 & 0.828 & 1.080 & 0.406 & 0.954 & 0.814 & 1.118 & 0.564 \\
\hline Alb & 0.247 & 0.080 & 0.763 & 0.015 & 0.1002 & 0.019 & 0.522 & 0.006 \\
\hline PNI & 0.910 & 0.832 & 0.995 & 0.038 & 0.868 & 0.772 & 0.975 & 0.017 \\
\hline GPS & 2.329 & 1.127 & 4.814 & 0.022 & 3.686 & 1.374 & 9.888 & 0.010 \\
\hline NLR & 1.139 & 0.955 & 1.358 & 0.149 & 1.236 & 1.004 & 1.523 & 0.046 \\
\hline PLR & 1.002 & 0.999 & 1.004 & 0.302 & 1.003 & 0.999 & 1.006 & 0.123 \\
\hline CAR & 3.284 & 0.958 & 11.26 & 0.059 & 8.445 & 1.396 & 51.09 & 0.020 \\
\hline
\end{tabular}

95\%CI: 95\% Confidence interval; HR: hazard ratio; DTC: differentiated thyroid carcinoma; OS: overall survival; PFS: progression-free survival; TP: treatment period; BMI: body mass index (kg/m2); Alb: serum albumin (g/dl); PNI: prognostic nutrition index; GPS: Glasgow prognostic score; NLR: neutrophil-to-lymphocyte ratio; PNR: platelet-to-lymphocyte ratio; CAR: CRP-albumin ratio.

(0, non-sarcopenia; 1, sarcopenia); BMI, body mass index $\left(\mathrm{kg} / \mathrm{m}^{2}\right)$; Alb, serum albumin (g/dl); PNI, prognostic nutrition index (no unit, only value); GPS, Glasgow prognostic score (no unit, just number); NLR, neutrophil-to-lymphocyte ratio (no unit, only value); PLR, platelet-to-lymphocyte ratio (no unit, only value); CAR, CRPalbumin ratio (no unit, only value).

Ethical consideration. The protocol for this investigation was approved by the Institutional Review Board of the Kanazawa University Hospital (2016-22, 2017-312).

\section{Results}

Patients' characteristics. Table I presents details regarding patients' background. SG included 9 patients, while NSG included 14 patients. Overall, 14 patients were diagnosed with radioiodine-refractory DTC, 4 patients were diagnosed with poorly DTC, and 5 patients were diagnosed with ATC. The body weight, body surface area, and BMI differed significantly between SG and NSG.
Efficacy for all patients. In SG the median OS was 12.8 months [95\% confidence interval $(\mathrm{CI})=4.9-28.7]$ while in NSG OS was not reached (NR) $(95 \% \mathrm{CI}=17.5-\mathrm{NR})$. In $\mathrm{SG}$ the median PFS was 11.6 months $(95 \% \mathrm{CI}=1.4-28.7)$ while in NSG PFS was not reached $(95 \% \mathrm{CI}=10.0-\mathrm{NR})$. The median TP in SG was 12.7 months $(95 \% \mathrm{CI}=4.5-20.7)$ while in NSG TP was not reached $(95 \% \mathrm{CI}=14.0-\mathrm{NR})$ (Table II). Figure 1 demonstrates the Kaplan-Meier curves of OS, PFS, and TP.

As for RR and DCR, there were no significant differences were observed between SG and NSG (Table III). Table IV presents the survival analyses based on nutrition indexes. Baseline albumin, PNI, and GPS significantly affected the survival time. Compared to patients presenting a lower PNI, patients with a higher PNI demonstrated better OS, PFS, and TP. In contrast, patients with higher GPS scores demonstrated a worse prognosis for OS, PFS, and TP.

Efficacy for DTC patients. In SG the median OS was 17.6 months $(95 \% \mathrm{CI}=11.6-\mathrm{NR})$, while in NSG this was not 

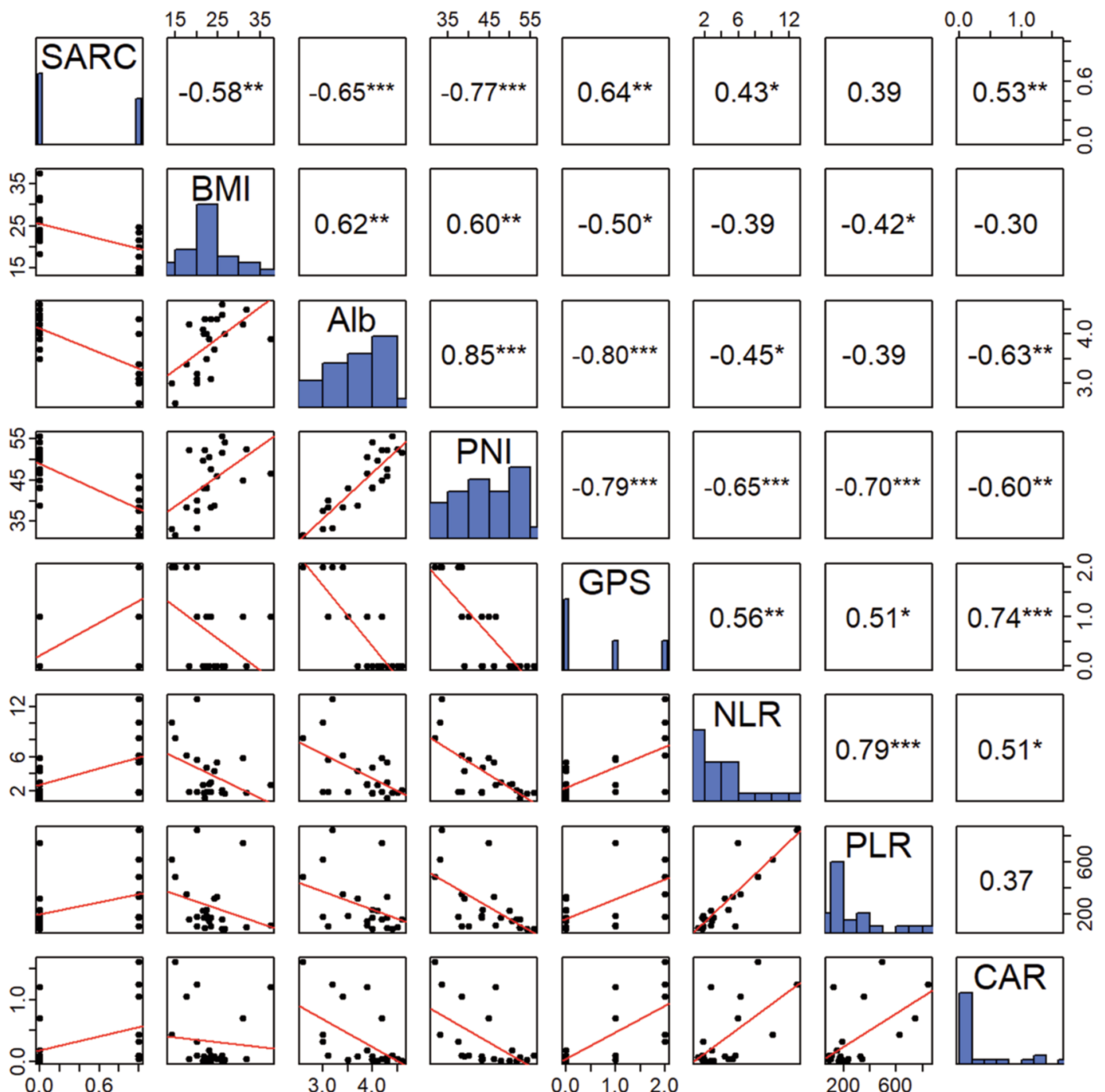

Figure 3. The correlation value of sarcopenia and several nutrition indexes. SARC: Sarcopenia; BMI: body mass index; Alb: albumin; PNI: prognostic nutrition index; GPS: Glasgow prognostic score; NLR: neutrophil-to-lymphocyte ratio; PLR: platelet-to-lymphocyte ratio; CAR: CRPalbumin ratio; $* p<0.05, * * p<0.01, * * * p<0.001$.

achieved (95\%CI=17.5-NR). In SG the median PFS was 12.8 months $(95 \% \mathrm{CI}=1.4-\mathrm{NR})$ while in NSG PFS was not reached (95\% CI=10.0-NR). The median TP in SG was 11.1 months $(95 \% \mathrm{CI}=3.3-\mathrm{NR})$ while in NSG this value was not attained (95\% CI=14.0-NR) (Table II). Figure 2 demonstrates the Kaplan-Meier curves of OS, PFS, and TP. In terms of RR and DCR, no significant differences were observed between SG and NSG (Table III).

Table IV shows the survival analysis based on nutrition indexes in DTC patients. Moreover, DTC patients presented results identical to all investigated patients.

Correlation matrix between sarcopenia and the other nutrition indexes. Figure 3 presents the correlation values for sarcopenia and other nutrition indexes. Based on these results, sarcopenia tended to correlate with nutritional markers overall. Particularly, sarcopenia demonstrated a strong correlation with albumin, PNI, and GPS. Additionally, albumin, PNI, and GPS were well correlated with one another. In contrast, sarcopenia demonstrated relatively weak correlation with NLR and PLR, as calculated from blood cell counts.

Safety. All patients were used for safety analysis. Patients in SG presented the higher incidence of infection and the worse grade of anemia, and nausea when compared to those in NSG. Conversely, patients in NSG demonstrated a worse grade of hand-foot syndrome (HFS) compared to those in SG (Figure 4). The grade of hematological toxicities, except for 


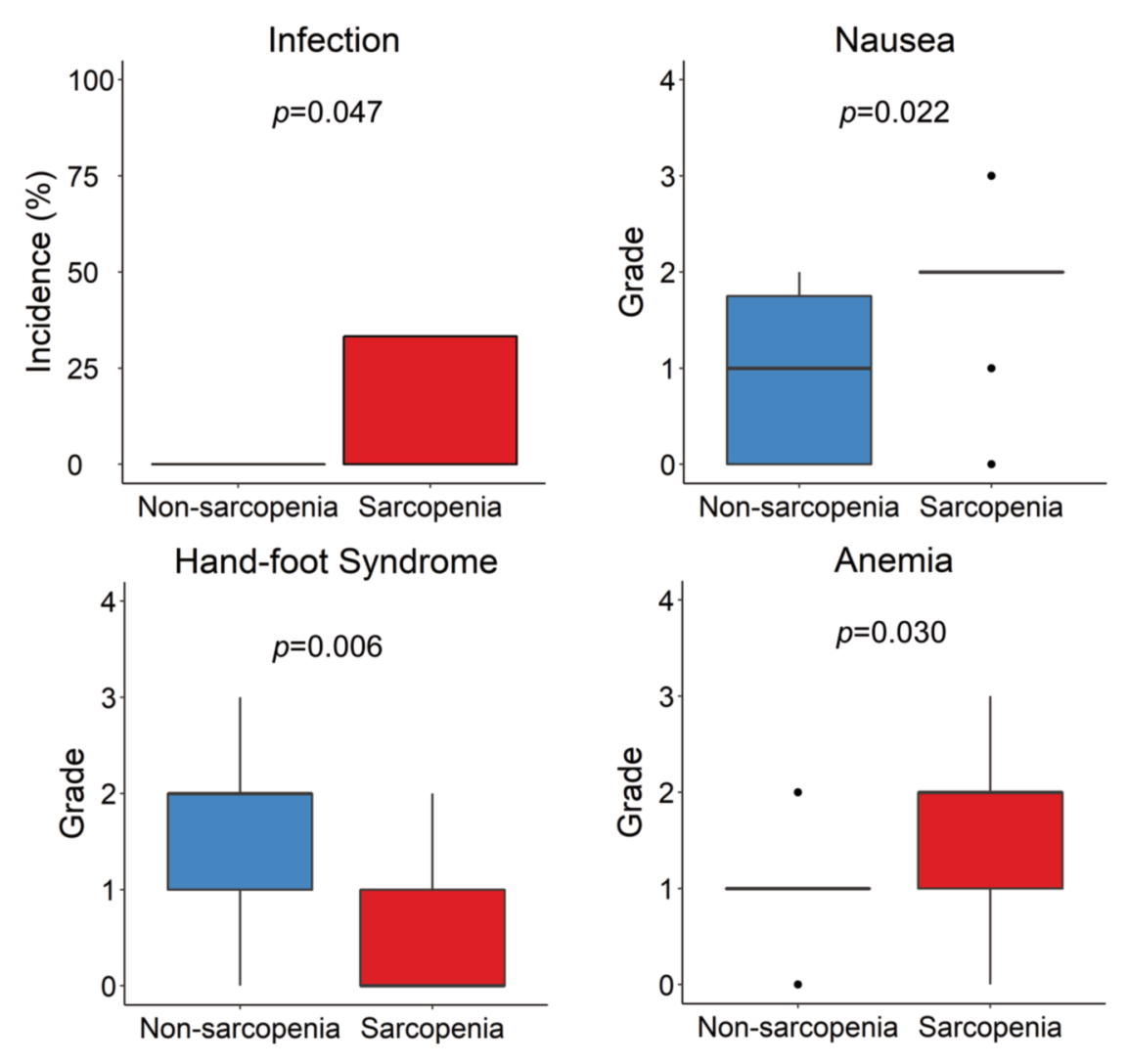

Figure 4. The adverse effects unique to the sarcopenia and non-sarcopenia groups.
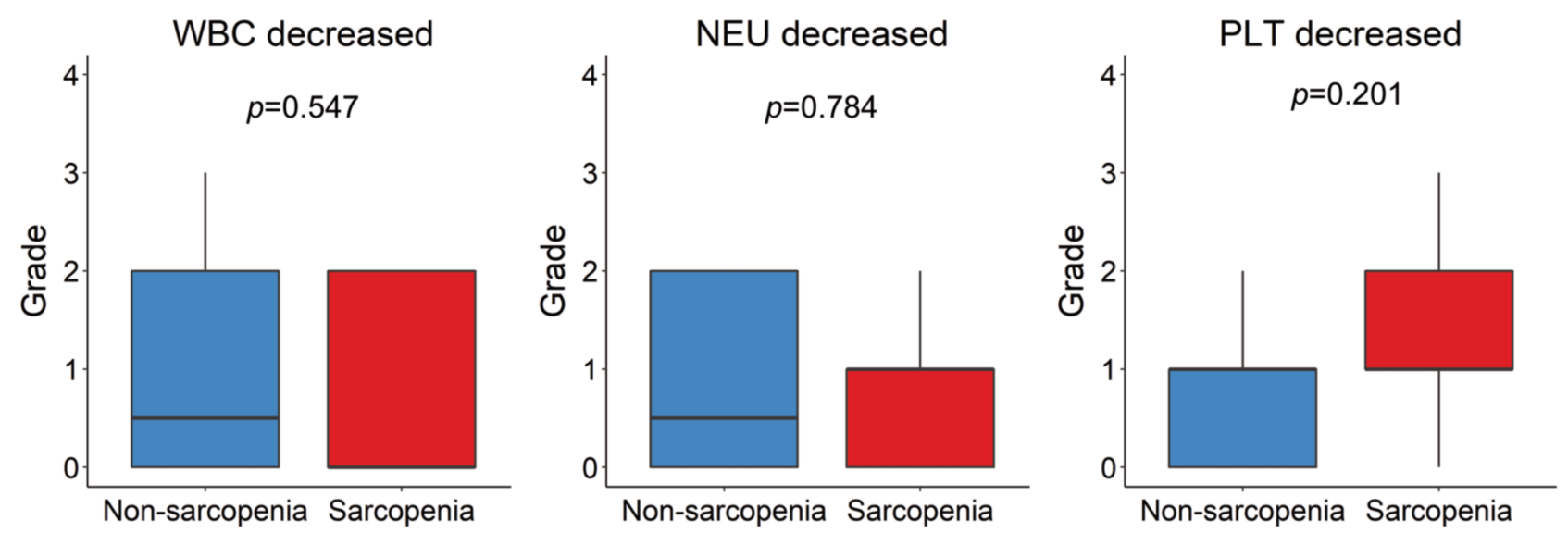

Figure 5. The hematological toxicities do not significantly differ between the sarcopenia group and non-sarcopenia groups. WBC: White blood cell; NEU: neutrophil; PLT: platelet.

anemia, and that of popular non-hematologic toxicities related to lenvatinib or sorafenib, such as proteinuria and hypertension, had no significant difference between SG and NSG. Moreover, the incidence of complications related to anti-angiogenesis agent, such as fistula, had no difference between SG and NSG (Figures 5 to 7).

\section{Discussion}

In the field of digestive cancer, nutrition assessment as a prognostic factor has been extensively reported. Recently, NLR, one strategy for nutrition assessment, has been reported as a prognostic factor in DTC patients undergoing molecular 

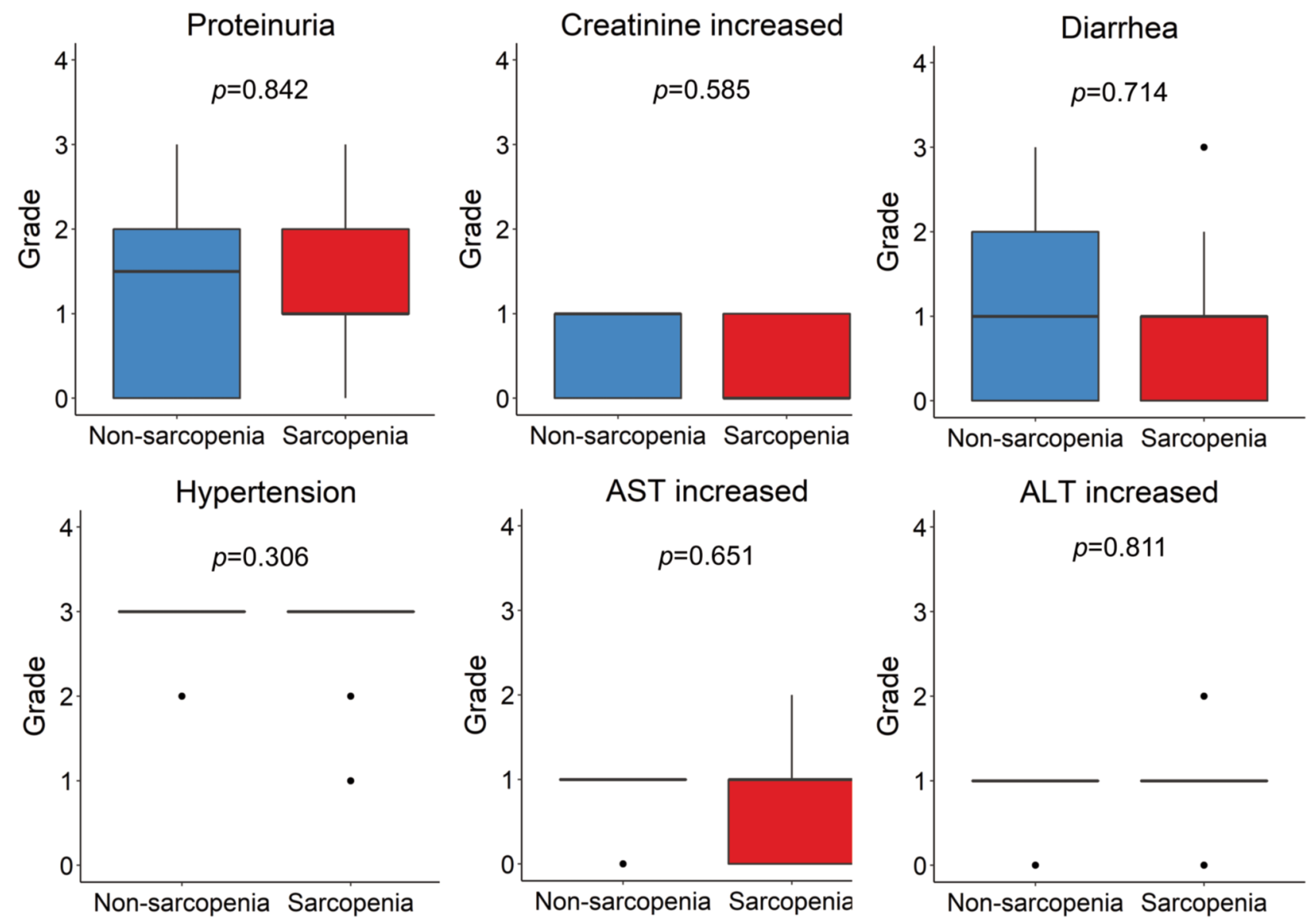

Figure 6. The non-hematological toxicities do not significantly differ between the sarcopenia and non-sarcopenia groups. AST: Aspirate aminotransferase; ALT: alanine aminotransferase.

targeted therapy (21). Moreover, patients with poorly DTC or ATC have demonstrated significantly higher NLR than DTC patients, and NLR could be used as a new diagnostic criterion to discriminate against aggressive thyroid cancers (22). Moreover, reports concerning thyroid cancer and nutrition assessment have been gaining momentum. As patients with advanced thyroid cancer can use fewer molecular targeted agents, it is crucial to determine the best time for drug introduction, ensuring both safety and effectiveness. Furthermore, in various digestive disorders including cancers, sarcopenia is reportedly associated with postoperative mortality, recurrence, and the development of adverse effects and complications $(23,24)$. Based on these reports, we investigated how sarcopenia, as well as the nutritional status before the initiation of molecular targeted therapy, may influence the prognosis and toxicity of molecularly targeted agents in patients with advanced thyroid cancer.

In this study, we obtained the following results. First, sarcopenia could be used as a prognostic and predictive factor for assessing the therapeutic effects of molecularly targeted agents in all patients with advanced thyroid cancer. Second, sarcopenia strongly correlated with albumin, PNI, and GPS, which could be additional prognostic factors. Third, DTC patients demonstrated the same trend as all other patients.

In the case of patients who develop RAI refractory differentiated cancer, it is important to decide when to introduce molecularly targeted agents in clinical settings. Based on the findings of this study, muscle mass and nutritional status before therapy could be associated with patient prognosis. According to the above results, as well as the report stating that nutritional intervention can improve the prognosis in patients with cancer $(25,26)$, patients should carefully monitor their muscle mass regularly, modulating their nutritional status from a medical perspective.

Interestingly, for molecularly targeted agents, profiles of $\mathrm{AE}$ differ depending on the presence or absence of sarcopenia. SG demonstrated a higher grade of anemia, infection, and nausea than NSG; conversely, NSG presented a higher grade of HFS than SG. As disease conditions in SG were worse compared to what was observed in NSG, it was considered that SG 

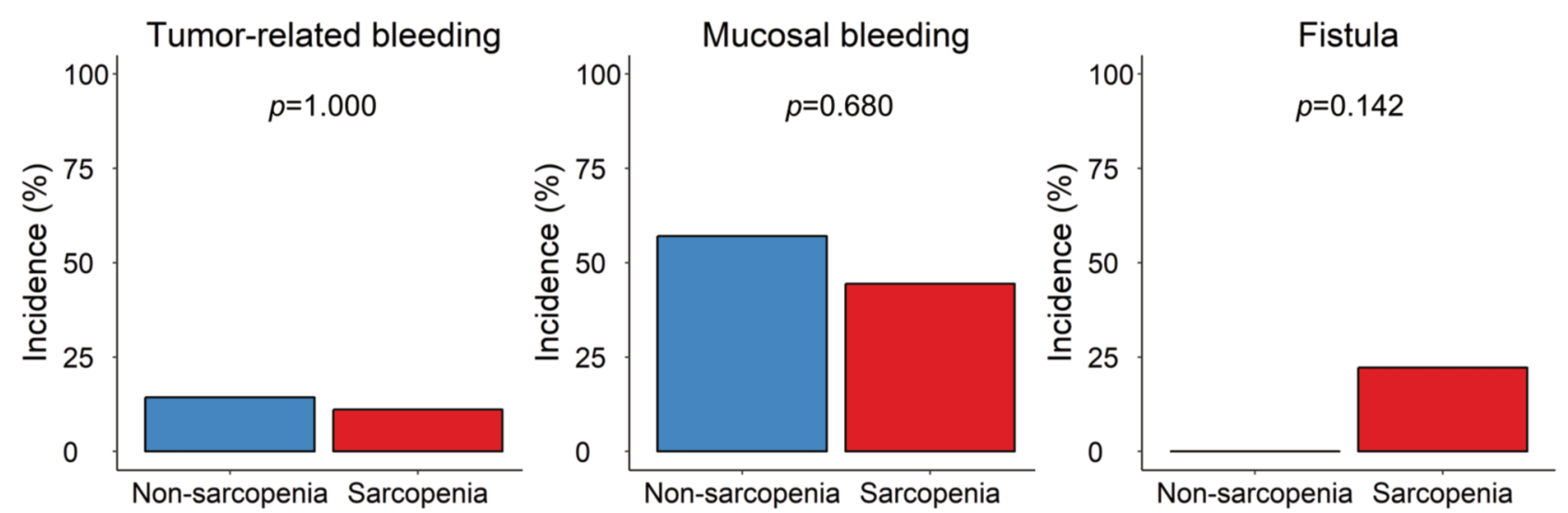

Figure 7. Complications related to molecular targeted therapy do not significantly differ between the sarcopenia and non-sarcopenia groups.

experienced anemia owing to exhaustion produced by advanced thyroid cancer. Furthermore, some reports suggest that sarcopenia possesses the risk to induce infection (27-30). Regarding the relationship between sarcopenia and nausea, we speculated that nausea induced a decline in food intake, resulting in malnutrition and sarcopenia.

NSG possessed sufficient muscle mass for active performance, consequently developing HFS easily. To understand this phenomenon from another standpoint, as SG demonstrated less muscle mass, muscle weakness, and low levels of physical activity, the palms and soles experienced less load, and hence, less HFS.

Additionally, no significant difference was observed between tumor responses in SG and NSG. Based on reports from the World Congress on Thyroid Cancer 3.5, in the SELECT trial (4), post hoc analysis on NLR presented no significant difference in RR between NLR $\leq 3$ and NLR $>3$, indicating that this result is consistent with the findings of the current study.

Some limitations related to this study include its retrospective nature, the small sample size, and the fact that sarcopenia is not common in clinical oncology. To resolve these limitations, we plan to conduct a prospective multicenter trial investigating the impact of sarcopenia on patient prognosis, as well as the adverse effect profile of molecular targeted therapy when introduced by physicians in patients with advanced thyroid cancer.

In conclusion, sarcopenia could be a prognostic factor in patients with advanced thyroid cancer receiving molecular targeted therapy. Therefore, routine evaluation of muscle mass and nutritional status may be beneficial in patients with advanced thyroid cancer undergoing molecular targeted therapy.

\section{Conflicts of Interest}

SY obtained a research grant from Eisai Inc. Other Authors have no potential conflicts of interest.

\section{Authors' Contributions}

YS and SY were involved with concept and design of this study and manuscript. YS, MF, AT and KO were involved with collecting data. YS and SY proofread the manuscript. All Authors have read and approved the final version of the edited manuscript.

\section{References}

1 Incidence rate of thyroid cancer in Japan. Available at: https://www.env.go.jp/chemi/rhm/h29kisoshiryo/h29kiso-03-0719.html [Last accessed 31th March 2017]

2 Prete A, de Souza PB, Censi S, Muzza M, Nucchi N and Sponziello M: Update on fundamental mechanisms of thyroid cancer. Front Endocrinol (Lausanne) 11: 102, 2020. PMID: 32231639. DOI: 10.3389/fendo.2020.00102

3 Brose MS, Nutting CM, Jarzab B, Elisei R, Siena S, Bastholt L, de la Fouchardiere C, Pacini F, Paschke R, Shong YK, Sherman SI, Smith JWA, Chung J, Kappeler C, Peña C, Molnár I, Schlumberger MJ and DECISION Investigators: Sorafenib in radioactive iodine-refractory, locally advanced or metastatic differentiated thyroid cancer: a randomised, double-blind, phase 3 trial. Lancet 384(9940): 319-328, 2014. PMID: 24768112. DOI: 10.1016/S0140-6736(14)60421-9

4 Schlumberger M, Tahara M, Wirth LJ, Robinson B, Brose MS, Elisei R, Habra MA, Newbold K, Shah MH, Hoff AO, Gianoukakis AG, Kiyota N, Taylor MH, Kim SB, Krzyzanowska MK, Dutcus CE, de las Heras B, Zhu J and Sherman SI: Lenvatinib versus placebo in radioiodine-refractory thyroid cancer. N Engl J Med 372(7): 621-630, 2015. PMID: 25671254. DOI: 10.1056/NEJMoa1406470

5 Davis L and Welth HG: Increasing incidence of thyroid cancer in the United States, 1973-2002. JAMA 295(18): 2164-2167, 2006. PMID: 16684987. DOI: 10.1001/jama. 295.18.2164

6 Nikiforova MN and Nikiforov YE: Molecular diagnostic and predictors in thyroid cancer. Thyroid 19(12): 1351-1361, 2009. PMID: 19895341. DOI: 10.1089/thy.2009.0240

7 Tahara M, Kiyota N, Yamazaki T, Chayahara N, Nakano K Inagaki L, Toda K, Enokida T, Minami H, Imamura Y, Sasaki T, Suzuki T, Fujino K, Dutcus CE and Takahashi S: Lenvatinib for anaplastic 
thyroid cancer. Front Oncol 7: 25, 2017. PMID: 28299283. DOI: 10.3389/fonc.2017.00025

8 McMillan DC: The systemic inflammation-based Glasgow Prognostic Score: a decade of experience in patients with cancer. Cancer Treat Rev 39(5): 534-540, 2013. PMID: 22995477. DOI: 10.1016/j.ctrv.2012.08.003

9 Huang DD, Zhou CJ, Wang SL, Mao ST, Zhou XY, Lou N, Zhang Z, Yu Z, Shen X and Zhuang C: Impact of different sarcopenia stages on the postoperative outcomes after radical gastrectomy for gastric cancer. Surgery 161(3): 680-693, 2017. PMID: 27712873. DOI: $10.1016 /$ j.surg.2016.08.030

10 Deng HY, Zha P, Peng L, Hou L, Huang KL and Li XY: Preoperative sarcopenia is a predictor of poor prognosis of esophageal cancer after esophagectomy: a comprehensive systematic review and mata-analysis. Dis Esophagus 32(3): doy115, 2019. PMID: 30496385. DOI: 10.1093/dote/doy 115

11 Hamaguchi Y, Kaido T, Okumura S, Kobayashi A, Shirai H, Yagi S, Kamo N, Okajima H and Uemoto S: Impact of skeletal muscle index, intramuscular adipose tissue content, and visceral to subcutaneous adipose tissue area ratio on early mortality of living donor liver transplantation. Transplantation 101(3): 565-574, 2017. PMID: 27926595. DOI: 10.1097/TP.0000000000001587

12 Vledder MGv, Levolger S, Ayez N, Verhoef C, Tran TCK and Ijzermans JNM: Body composition and outcome in patients undergoing resection of colorectal liver metastases. Br J Surg 99(4): 550-557, 2012. PMID: 22246799. DOI: 10.1002/bjs.7823

13 Yoshizumi T, Shirabe K, Nakagawa H, Ikegami T, Harimoto N, Toshima T, Yamashita Y, Ikeda T, Soejima Y and Mehara Y: Skeletal muscle area correlates with body surface area in healthy adults. Hepatol Res 44(3): 313-318, 2014. PMID: 23607375. DOI: 10.1111/hepr.1211

14 Forrest LM, McMillan DC, McArdle CS, Angerson WJ and Dunlop DJ: Evaluation of cumulative prognostic scores based on the systemic inflammatory response in patients with inoperable nonsmall-cell lung cancer. Br J Cancer 89(6): 1028-1030, 2003. PMID: 12966420 . DOI: 10.1038/sj.bjc.6601242

15 Kanda M, Fujii T, Kodera Y, Nagai S, Takeda S and Nakao A: Nutritional predictors of postoperative outcome in pancreatic cancer. Br J Surg 98(2): 268-274, 2011. PMID: 20960457. DOI: 10.1002/bjs. 7305

16 Chiang SF, Hung HY, Tang R, Changchien CR, Chen JS, You YT, Chiang JM and Lin JR: Can neutrophil-tolymphocyte ratio predict the survival of colorectal cancer patients who have received curative surgery electively? Int J Colorectal Dis 27(10): 1347-1357, 2012. PMID: 22460305. DOI: 10.1007/s00384-012-1459-x

17 Wu Y, Li C, Zhao J, Yang L, Liu F, Zheng H, Wang Z and Xu Y: Neutrophil-to-lymphocyte and platelet-to-lymphocyte ratios predict chemotherapy outcomes and prognosis in patients with colorectal cancer and synchronous liver metastasis. World J Surg Oncol 14(1): 289, 2016. PMID: 27852294. DOI: 10.1186/s12957-016-1044-9

18 Ishizuka M, Nagata H, Takagi K, Iwasaki Y, Ghibuya N and Kubota K: Clinical significance of the $\mathrm{C}$-reactive protein to albumin ratio for survival after surgery for colorectal cancer. Ann Surg Oncol 23(3): 900-907, 2016. PMID: 26530445. DOI: 10.1245/s10434-015-4948-7

19 Eisenhauer EA, Therasse P, Bogaerts J, Schwartz LH, Sargent D, Ford R, Dancey J, Arbuck S, Gwyther S, Mooney M, Rubinstein L, Shankar L, Dodd L, Kaplan R, Lacombe D and Verweij J: New response evaluation criteria in solid tumours: revised RECIST guideline (version 1.1). Eur J Cancer 45(2): 228-247, 2009. PMID: 19097774. DOI: 10.1016/j.ejca.2008.10.026
20 Common Terminology Criteria for Adverse Events (CTCAE) v5.0. Available at: https://ctep.cancer.gov/protocolDevelopment/electronic_ applications/ctc.htm\#ctc_50 [Last accessed $4^{\text {th }}$ October 2020]

21 Ozmen S, Timur O, Calik I, Altinkaynak K, Simsek E, Gozcu H, Arslan A and Carliglu A: Neutrophil-lymphocyte ratio (NLR) and platelet-lymphocyte ratio (PLR) may be superior to C-reactive protein (CRP) for predicting the occurrence of differentiated thyroid cancer. Endocr Regul 51(3): 131-136, 2017. PMID: 28858848. DOI: 10.1515/enr-2017-0013

22 Cho JS, Park MH, Ryu YJ, Yoon JH: The neutrophil to lymphocyte ratio can discriminate anaplastic thyroid cancer against poorly or well differentiated cancer. Ann Surg Treat Res 88(4): 187-192, 2015. PMID: 25844352. DOI: 10.4174/astr.2015.88.4.187

23 Nishikawa H, Yoh K, Enomoto H, Iwata Y, Sakai Y, Kishino K, Shimono Y, Ikeda N, Takashima T, Aizawa N, Takata R, Hasegawa $\mathrm{K}$, Koriyama T, Yuri Yukihisa, Nishimura T, Nishiguchi S and Iijima H: Sarcopenia and frailty in chronic liver damage: common and different points. In Vivo 34(5): 2549-2559, 2020. PMID: 32871784. DOI: 10.21873 /invivo. 12072

24 Hsueh SW, Liu KH, Hung CY, Tsai CY, Hsu JT, Tsang NM, Hsueh WH, Yang $\mathrm{C}$ and Chou WC: Predicting postoperative events in patients with gastric cancer: a comparison of five nutrition assessment tools. In Vivo 34(5): 2803-2809, 2020. PMID: 32871818. DOI: 10.21873 /invivo.12106

25 Qiu Y, You J, Wang K, Cao Y, Hu Y, Zhang H, Fu R, Sun Y, Chen $\mathrm{H}$, Yuan L and Lyu Q: Effect of whole-course nutrition management on patients with esophageal cancer undergoing concurrent chemoradiotherapy: A randomized control trial. Nutrition 69: 110558, 2020. PMID: 31526964. DOI: 10.1016/j.nut.2019.110558

26 Van Blarigan EL, Fuchs CS, Niedzwiecki D, Zhang S, Saltz LB, Mayer RJ, Mowat RB, Whittom R, Hantel A, Benson A, Atienza D, Messino M, Kindler H, Venook A, Ogino S, Giovannucci EL, $\mathrm{Ng} \mathrm{K}$ and Meyerhardt JA: Association of survival with adherence to the American Cancer Society nutrition and physical activity guidelines for cancer survivors after colon cancer diagnosis: The CALGB 89803/Alliance trial. JAMA Oncol 4(6): 783-790, 2018. PMID: 29710284. DOI: 10.1001/jamaoncol.2018.0126

27 Babu JM, Kalagara S, Durand W, Antoci V, Deren ME and Cohen E: Sarcopenia as a risk factor for prosthetic infection after total hip or knee arthroplasty. J Arthroplasty 34(1): 116-122, 2019. PMID: 30337254. DOI: $10.1016 / j$.arth.2018.09.037

28 Takagi K, Yoshida R, Yagi T, Umeda Y, Nobuoka D, Kuise T and Fujiwara T: Radiographic sarcopenia predicts postoperative infectious complications in patients undergoing pancreaticoduodenectomy. BMC Surg 17(1): 64, 2017. PMID: 28549466. DOI: 10.1186/s12893017-0261-7

29 Fujikawa H, Araki T, Okita Y, Kondo S, Kawamura M, Hiro J, Toiyama Y, Kobayashi M, Tanaka K, Inoue Y, Mohri Y, Uchida K and Kusunoki M: Impact of sarcopenia on surgical site infection after restorative proctocolectomy for ulcerative colitis. Surg Today 47(1): 92-98, 2017. PMID: 27255541. DOI: 10.1007/s00595-016$1357-\mathrm{X}$

30 Lieffers JR, Bathe OF, Fassbender K, Winget M and Baracos VE: Sarcopenia is associated with postoperative infection and delayed recovery from colorectal cancer resection surgery. Br J Cancer 107(6): 931-936, 2012. PMID: 22871883. DOI: 10.1038/bjc.2012.350

Received August 29, 2020

Revised October 10, 2020

Accepted October 16, 2020 